\title{
Un Enfoque Multiobjetivo para la Asignación de Canales en Sistemas Celulares
}

\author{
Héctor E. Carranza ${ }^{1^{*}}$, Luis Chávez ${ }^{2}$, María L. Fissore ${ }^{1}$ y Silvia B. Simón ${ }^{1}$ \\ Universidad Nacional de Río Cuarto, (1) Facultad de Ingeniería, (2) Facultad de Ciencias Exactas, \\ Fisico Químicas y Naturales Campus Universitario, (5800) Río Cuarto-Argentina \\ (e-mail: \{hcarranza, mfissore, ssimón\}@ing.unrc.edu.ar, Ichavez@dc.exa.unrc.edu.ar)
}

*autor a quien debe ser dirigida la correspondencia

\section{Resumen}

En este trabajo se propone un algoritmo de asignación de canales para reducir la probabilidad de bloqueo de sistemas celulares y ampliar la capacidad del sistema para absorber nuevas llamadas. El algoritmo debe proveer un conjunto de soluciones de compromiso entre dos objetivos contrapuestos; por un lado minimizar la cantidad de reasignaciones y por el otro minimizar el nivel de interferencia debido a la flexibilización de las restricciones de compatibilidad electromagnética. Se propone un enfoque heurístico basado en un paradigma evolutivo para resolver el problema de optimización multiobjetivo. Para que la evolución sea eficiente, se incorpora en el proceso evolutivo conocimiento específico del problema. Dos algoritmos evolutivos multiobjetivo (SPEAll y NSGA II) son comparados usando dos diferentes métricas. Los resultados muestran que la metodología propuesta puede ser aplicada a las diferentes generaciones de sistemas de comunicaciones móviles.

\section{A Multiobjective Approach for Cellular Systems Channel Assignment}

\begin{abstract}
To improve the quality of service in cell systems, techniques of Dynamic Assignment Channels are used to reduce the probability of blocking calls. In this paper an algorithm of assignment channels is required to reduce the probability of blocking, extending the system capacity to add new calls. This is obtained by using an interchange of channels of current calls and reducing the restrictions of electromagnetic compatibility. According to this, the algorithm has to provide solutions of commitments between two different objectives; on the one hand reduce the amount of assignments, and on the other to do the same with the level of interference due to the flexible restrictions of electromagnetic compatibility. A heuristic focus based on the evolution paradigm is presented to solve the multi-objective optimization problem. Two evolutionary multi-objective algorithms (SPEAll y NSGA II) are compared by the use of two different metrics. The results show that the proposed methodology can be applied to different generations of mobile communication systems.
\end{abstract}

Keywords: cellular networks, channel assignment, evolutionary algorithms, optimization 


\section{INTRODUCCIÓN}

En los sistemas celulares el área de cobertura de servicio es dividida en celdas, en cada una de las cuales se ubica una estación base. Dependiendo del método de acceso al medio utilizado, los sistemas pueden ser clasificados en sistemas canalizados y no canalizados. Dentro de los primeros se distinguen como métodos de acceso, TDMA, FDMA o combinaciones de ambos. En estos casos el término canal es usado para identificar una porción del espectro en FDMA, o para identificar una porción de tiempo (time spot) en TDMA (Whenhao et al., 2003). En adelante se analizará el caso de sistemas celulares cuyo método de acceso es FDMA.

El tráfico en sistemas celulares es generalmente alto por lo que, para resolver más de una llamada simultánea en distintas celdas, es necesaria la reutilización de canales. Esta reutilización provoca interferencias con la consiguiente degradación de la calidad de las comunicaciones. De modo que, para mantener dicha calidad, se deben imponer ciertas restricciones sobre los canales que pueden ser reutilizados en forma simultánea dentro de un área. Esto se logra mediante la determinación de bandas de guardas lo que trae aparejado una reducción de la eficiencia en el uso del espectro. Los métodos para la asignación de canales deben ajustarse a tres tipos de restricciones de compatibilidad electromagnéticas RCE (Sivarajan, 1989), es decir:

Restricciones Cocanal: el mismo canal no puede ser asignado simultáneamente a ciertos pares de celdas.

Restricciones de canal adyacente: canales muy próximos en frecuencia no pueden ser asignados simultáneamente a celdas con cierta proximidad geográfica.

Restricciones Co-sitio: canales asignados simultáneamente en la misma celda deben guardar cierta separación en frecuencia.

Considerando que los requerimientos de tráfico son cada vez mayores se plantea la necesidad de contar con esquemas de asignación de canales eficientes. Islam y Hossain (2004), propusieron un algoritmo, basado en coloreo de grafos, para optimizar la asignación de canales respetando las RCE a partir de la topología de la red representada por un grafo equivalente, el vector de demanda, la matriz de interferencias y la lista de canales. Naik y Wei (2004), han propuesto una metodología basada en el concepto de llamada en espera para mejorar el desempeño de los algoritmos de asignación dinámica de canales en redes celulares. Su trabajo está basado en la hipótesis de que el impacto sobre la calidad de servicio por causa de la espera en una nueva llamada es menor que el causado por un bloqueo de la misma. Vidyarthi et al (2005), utilizaron una técnica basada en estrategias evolutivas para resolver el problema de asignación dinámica de canales, minimizando la cantidad de reasignaciones realizadas con las RCE como restricciones a satisfacer. Fu et al. (2003), propusieron un algoritmo de asignación de canales que utiliza tres etapas, las cuales se aplican sucesivamente hasta lograr la resolución de la asignación. En primer lugar se define un intervalo para las asignaciones de las frecuencias en función del análisis de las restricciones de compatibilidad electromagnética. La segunda etapa implementa un procedimiento del tipo goloso para incorporar celdas adyacentes. En la última etapa un I algoritmo genético se encarga de buscar el ordenamiento óptimo de las llamadas en las celdas en las cuales no hay asignaciones realizadas. El problema del ordenamiento de las llamadas es atacado aplicando un índice de ordenamiento de las celdas (o regiones) según la dificultad de realizar asignaciones en las mismas. Lima et al. (2002), implementaron un algoritmo evolutivo con representación binaria para la matriz de asignación de canales y modelaron el problema con restricciones basadas en la distancia de reúso. La función de costo es una combinación ponderada de diferentes índices que tienen en cuenta el uso de los canales en las celdas co-canales de distintos niveles, a la vez que consideran la cantidad de canales bloqueados.

En el presente trabajo se propone analizar el impacto en la calidad de servicio al considerar dos alternativas para evitar el bloqueo de una llamada entrante o la desconexión de una llamada en curso: ordenar las llamadas preexistentes o bien permitir cierta violación de las restricciones de compatibilidad electromagnética. Ambas alternativas producen una disminución de la calidad de servicio: la primera debido a las reasignaciones que deben realizarse y la segunda porque se generan interferencias. 
Es conveniente tanto realizar el mínimo número posible de reasignaciones como minimizar la violación de las restricciones de compatibilidad. De hecho, si estas últimas son respetadas rigurosamente será necesario realizar mayor cantidad de reasignaciones para alojar la nueva llamada. En la otra parte, si no se permite realizar ninguna reasignación se debería aceptar un mayor grado de violación de las RCE. Minimizar el número de reasignaciones al mismo tiempo que se minimiza el nivel de interferencias conduce a la resolución de un problema de optimización que puede ser analizado como un problema multiobjetivo. Se propone una alternativa para poder atender el mayor tráfico posible a través de las acciones descriptas mediante la resolución de un problema multiobjetivo resuelto a través de un enfoque evolutivo. Se evalúan 2 algoritmos genéticos multiobjetivo: SPEA II y NSGA II y se definen algunas métricas para comparar el desempeño de los mismos para resolver el problema planteado.

\section{DETERMINACIÓN DE LAS FUNCIONES OBJETIVO}

El esquema planteado requiere la definición de dos funciones de costo, una para cada objetivo, de tal manera de obtener una medida tanto del deterioro de la calidad de servicio debido al aumento de interferencias como de la degradación de la calidad ocasionada por las maniobras de reasignación.

\section{Análisis de interferencias}

Un esquema de asignación puede ser expresado en los siguientes términos:

Sean:

z: El número de canales disponibles.

$n$ : El número de celdas.

d: El último vector de demandas con $n$ componentes en el cual cada componente $d_{i}$ del vector d corresponde al último número de frecuencias requeridas en la celda $i$.

C: La matriz de compatibilidad electromagnética $n \times n$ en la cual cada elemento $c_{i, j}$ describe la mínima separación de frecuencias usadas simultáneamente en las celdas $i$ y $j$.

Entonces el esquema es representado mediante una matriz de asignaciones $\mathbf{F}(n \times z)$ en la cual cada elemento $f_{i, j}$ es definido como:

$$
f_{i j}=\left\{\begin{array}{c}
1 \text { si la } j \text {-ésima frecuencia se asigna } \\
\text { a la } i \text {-ésima celda } \\
0 \text { en otro caso }
\end{array}\right.
$$

La restricción de compatibilidad electromagnética puede ser expresada de la siguiente manera:

$$
\begin{aligned}
\sum_{j=1}^{z} f_{i j} \geq d_{i} & \text { para } 1 \leq i \leq n \\
|p-q| \geq c_{i j} & \text { para } 1 \leq p, q \leq z \text { y } 1 \leq i, j \leq n \\
& \text { tal que } f_{i p}=f_{j q}=1
\end{aligned}
$$

Cuando son asignados los canales para atender el tráfico, respetando lo indicado por una matriz de compatibilidad electromagnética $\mathbf{C}$, no existe interferencia entre los usuarios. Por el contrario, si la asignación se realiza sin respetar las RCE, el daño a la calidad de servicio puede ser representado mediante una matriz de interferencia IT, de dimensión $n \times z$, cuyos elementos it $t_{i j}$ aproximan la interferencia, en el canal $j$ de la celda $i$, generada por el resto de los canales en todas las celdas del sistema. Para la determinación del valor de los elementos de la matriz interferencia se tomarán en cuenta dos situaciones: la interferencia provocada por los canales utilizados en la misma celda y la interferencia provocada por los canales utilizados en otras celdas. Para la primera situación se utiliza el perfil del filtro del receptor del móvil.

Para conocer los canales utilizados en cada celda se observan las filas de la matriz de asignación $\mathbf{F}$ $y$, para cada canal se calcula la suma de las interferencias provocadas por los otros canales 
utilizados en la misma celda. Partiendo del perfil del filtro se determina la atenuación correspondiente a una separación en el espectro de un canal (una octava), que es de $24 \mathrm{~dB}$. Con este cálculo se determinan los elementos de una matriz denominada matriz de Interferencia Co-Sitio (ICS) cuyos elementos están dados por la siguiente expresión:

$i c s_{h j}=\sum_{\substack{i=1 \\ i \neq j}}^{z}\left(\frac{f_{h, i}}{(\text { Atenuacion por octava })^{|j-i|}}\right)$

\section{Donde:}

$i c s_{h, j}$ : interferencia en el canal $j$ de la celda $h$

$f_{h, i}:$ elementos $h_{i}$ de la matriz de asignación

$|j-i|$ : separación entre los canales $i$ y $j$ medida en cantidad de canales.

$1 \leq h \leq n$

$1 \leq j \leq z$

Para la determinación de la interferencia provocada por los canales utilizados en otras celdas se toma en cuenta la distancia $d c_{i j .}$ entre las celdas $i$ y $j$ medidas en cantidad de radios de celda. A partir de la matriz de asignación $\mathbf{F}$ se determinan los elementos de una matriz denominada matriz de Interferencia Co-Canal (ICC) cuyos elementos están dados por la siguiente expresión:

$$
i c c_{h j}=\sum_{\substack{i=1 \\ i \neq h}}^{n} \frac{f_{i j}+i c s_{i j}}{\left(d c_{i h}-1\right)^{\gamma}}
$$

Donde:

$i c c_{h, j}$ : interferencia cocanal en el canal $j$ de la celda $h$

$i c s_{h, j}$ : elemento $i, j$ de la matriz de interferencia cositio con $1 \leq h \leq n \quad$ y $\quad 1 \leq j \leq z$

$\gamma$ Coeficiente de atenuación de camino libre (tipicamente $\gamma=4$ )

$d c_{i, h}$ : distancia entre las celdas $i$ y $h$ medida en cantidad de radios de celda

La suma IT=ICS+ICC de ambas matrices representa el monto total de interferencias

En un escenario de funcionamiento real es deseable minimizar el valor promedio de interferencias así como asegurar una distribución apretada alrededor de dicho valor. Por lo tanto la función de costo por interferencias se expresa de la siguiente manera:

Costo por interferencia $=\alpha . \sigma+\beta . \mu ;$ con $0 \leq \alpha, \beta \leq 1)$

Donde $\alpha$ y $\beta$ son parámetros de ponderación, ajustables por el usuario mientras que $\mu$ y $\sigma$ son respectivamente la media y desvío estándar de los $i t_{i j}$ tal que $f_{i j}=1$

\section{Análisis de reasignaciones}

Al generarse una solicitud de asignación para una nueva llamada, que no pueda ser atendida en la situación de asignaciones preexistente sin violar las RCC, se resolverá el problema cambiando el esquema de las asignaciones anteriores.

Dados una $\mathbf{F}_{\text {inicial }}$ y una celda $c$, demandante, se busca $\mathbf{F}_{\text {salida }}$ tal que a partir de $\mathbf{F}_{\text {salida }}$ se pueda asignar el tráfico solicitado por $c$, respetando las restricciones indicadas por $\mathbf{C}$ y, además la cantidad de cambios de asignación entre $\mathbf{F}_{\text {inicial }}$ y $\mathbf{F}_{\text {salida }}$ sea mínima. Esta cantidad de reasignaciones puede ser expresada como:

Nreasig $=\frac{G\left(\text { Xor }\left(\mathbf{F}_{\text {inicial }}, \mathbf{F}_{\text {salida }}\right)\right)}{2}$

Donde $G(\mathbf{M})$ es una función que cuenta la cantidad de unos en una matriz binaria $\mathbf{M}$. 
El modelado del problema multiobjetivo planteado puede expresarse como sigue:

$$
\begin{array}{ll}
\min & \alpha \cdot \mu+\beta \cdot \sigma \\
\min & \frac{G\left(\operatorname{Xor}\left(\mathbf{F}_{\text {inicial }}, \mathbf{F}_{\text {salida }}\right)\right)}{2}
\end{array}
$$

\section{CARACTERÍSTICAS DE LOS ALGORITMOS SELECCIONADOS}

SPEA II: es un algoritmo evolutivo multiobjetivo con elitismo. La población inicial, la representación de las soluciones y operadores evolutivos son estándares: distribución uniforme, representación binaria, selección por torneo binario, cruzamiento de un punto, mutación bit-flip. El poder real de SPEA II radica en la operación de preservación del elitismo. Se crea un conjunto externo (archivo) para almacenar soluciones previas no dominadas el cual es combinado con la población actual para formar el próximo archivo que es entonces usado para crear descendientes para la próxima generación. El tamaño del archivo es fijo, puede ser del mismo tamaño de la población. Por lo tanto, existen dos situaciones especiales cuando se pasan soluciones al archivo. Si el número de soluciones no dominadas es menor que el tamaño del archivo, éste se completa con otras soluciones dominadas tomadas de la población. La selección se realiza de acuerdo a un valor de aptitud definido específicamente por SPEA. La segunda situación sucede cuando el número de soluciones no dominadas supera el tamaño del archivo. En este caso, se aplica un operador de truncamiento. La solución que tiene la menor distancia a otras soluciones es removida del conjunto.

NSGA II: también es un algoritmo con elitismo. La principal diferencia con SPEA II radica en la operación de preservación de elitismo. El tamaño del archivo es igual al de la población inicial. El archivo actual es determinado sobre la base de la combinación de la población actual y el archivo previo. Para ello, NSGA II aplica un ranking de dominancia para clasificar la población en un número de niveles, de modo tal que el primer nivel es el mejor nivel en la población. El archivo se crea basado en el orden de rango de los niveles: el mejor rango se selecciona primero. Si el número de individuos en el archivo es menor que el tamaño de la población, se toma en cuenta el próximo nivel. Si agregar un nuevo nivel incrementa el número de individuos en el archivo excediendo el tamaño de la población inicial, se aplica un operador de truncamiento a dicho nivel basado en la distancia de apiñamiento (crowding). El operador de truncamiento remueve el individuo con menor distancia de apiñamiento. Luego a partir del archivo se crea una población de descendientes del mismo tamaño que la población inicial usando selección por torneo sobre la base de la distancia de apiñamiento. La regla de selección establece que cuando hay dos soluciones con el mismo rango, la ganadora es la que tiene mayor valor de distancia de apiñamiento.

\section{MÉTRICAS DE DESEMPEÑO}

La valoración del desempeño de los optimizadores multi-objetivo debería tomar en cuenta al menos los siguientes dos criterios: la distancia al frente óptimo de Pareto de las soluciones obtenidas y la distribución de dichas soluciones. Para la medición de estos aspectos, en la literatura se han propuesto varias métricas (Deb, 2001; Deb et al., 2002; Srinivas, 1994, Zitzler et al., 2001). Algunas de las métricas requieren el conocimiento de las verdaderas soluciones Pareto óptimas, las cuales para el problema planteado son desconocidas. Teniendo en cuenta esta limitación, se eligieron las siguientes tres mediciones: 1) tamaño del espacio dominado 2) cobertura de dos frentes de Pareto, y 3) no uniformidad del frente de Pareto. Las primeras dos métricas miden la convergencia de las soluciones Pareto, mientras que la última mide la distribución de las soluciones Pareto. Una descripción detallada de conceptos y técnicas de optimización multiobjetivo utilizando algoritmos evolutivos puede encontrarse en Coello et al. (2002).

\section{Tamaño del espacio dominado}

El tamaño del espacio dominado S(A) es una medida del tamaño del espacio objetivo que es cubierto por el conjunto no dominado A. Como un ejemplo, el tamaño del espacio dominado se ilustra en la Figura 1. 


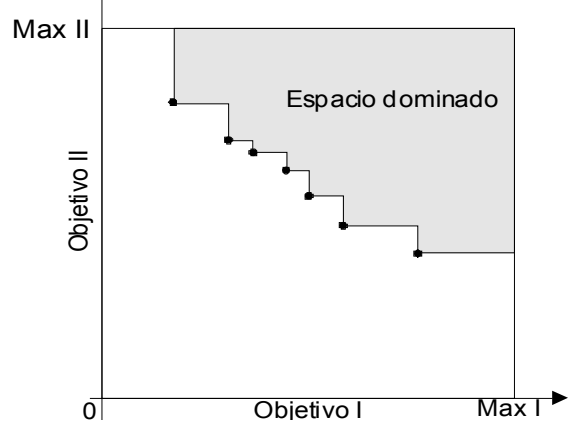

Fig. 1: Espacio dominado por un conjunto de Pareto cuando dos objetivos son minimizados

Dado el problema de optimización involucra la minimización de dos objetivos, para determinar el tamaño del espacio dominado, se elige un valor razonable máximo para cada objetivo (max I y maxII). Mayores valores de $S(A)$ indican mejor desempeño

\section{Cobertura de dos frentes Pareto}

Esta medición compara dos conjuntos de Pareto uno con otro. Dados dos conjuntos de Pareto $A$ y $B$, la cobertura $C(A, B)$ asigna al par ordenado $(A, B)$ un valor en el intervalo [0, 1]:

$C(A, B)=\frac{|\{b \in B I \exists a \in A: a \succ b\}|}{|B|}$

Donde $|B|$ indica el número de soluciones en el conjunto $B$. Si $C(A, B)=1$ entonces todos los individuos de $B$ son dominados por $A$. Por el contrario, si $C(A, B)=0$ entonces ningún individuo en $B$ es dominado por $A$. Si $C(A, B)>C(B, A)$ esto significa que el conjunto $A$ tienen mejores soluciones que el conjunto $B$.

\section{No uniformidad del Frente Pareto}

Para medir de no uniformidad de la distribución de un frente de Pareto, se introduce el valor $D(A)$ dado por la distribución de la distancia Euclideana $\left(d_{i}\right)$ entre dos puntos consecutivos (soluciones) a lo largo del frente de Pareto:

$D(A)=\sqrt{\frac{\sum_{i}\left(d_{i} / \bar{d}-1\right)^{2}}{|A|-1}}$

Cuando $D(A)=0$, el espaciado en el frente de Pareto es uniforme. Mientras mayor sea el valor de $D(A)$ mayor es la no uniformidad del frente. Por lo tanto se desearán bajos valores de $D(A)$.

\section{RESULTADOS Y DISCUSIÓN}

Para la evaluación de los experimentos se planteó un sistema celular de 25 celdas y 76 canales disponibles. Se consideró el siguiente vector de demandas actuales (Wan, 1996):

$\mathbf{d}=(10,11,9,5,9,4,5,7,4,8,8,8,10,7,7,6,4,5,5,7,6,4,5,7,5)$

Dichas demandas han sido satisfechas mediante una asignación de canales especificada por una matriz $\mathbf{F}_{\text {inicial. }}$ A partir de esa situación actual se supuso el ingreso al sistema una nueva solicitud de canal en la celda número 12.

La Tabla 1 muestra los valores de los parámetros y operadores comunes usados para los algoritmos evaluados.

Se ejecutaron 10 ejecuciones independientes para cada algoritmo. NSGA II encontró 34 soluciones mientras que SPEA II encontró 19 soluciones sobre un total de 100 individuos. 
Tabla 1: Valores para los parámetros de los algoritmos multiobjetivo

\begin{tabular}{lc}
\hline \hline \multicolumn{1}{c}{ Parámetro } & Valor \\
\hline Tamaño de Población & 100 \\
Generaciones & 200 \\
Probabilidad de Cruzamiento & 0.8 \\
Probabilidad de Mutación & 0.001 \\
Selección & Torneo binario \\
Cruzamiento & Un punto \\
Mutación & bit-flip. \\
\hline \hline
\end{tabular}

La calidad de las soluciones obtenidas es medida mediante las métricas de calidad $S(A), D(A)$, y $\mathrm{C}(A, B)$. Para el cálculo de la métrica $S(A)$, los valores máximos elegidos para cada objetivo son $\max _{1}=0,35$ y $\max _{2}=36$. NSGA II conduce a un mayor valor de $S(A)$ indicando un mejor desempeño a favor de este método. Sin embargo, esta métrica no puede garantizar que un frente domine enteramente a otro cuando las curvas se cruzan como en el caso bajo estudio. Además esta métrica no aporta información acerca de la distribución de las soluciones. Por esta razón se aplicó además la métrica $D$. Los valores de la métrica $D$ muestran que el espaciado de las soluciones de NSGAll es más uniforme que las soluciones de SPEA II. La tabla 2 permite una observación comparativa de ambas métricas.

Tabla 2: Valores para las métricas $S(A)$ y $D(A)$

\begin{tabular}{lccc}
\hline \hline Algoritmo & Soluciones & S(A) & D $(A)$ \\
\hline SPEA II & 19 & 8,963 & 3,276 \\
NSGAII & 34 & 9,487 & 1,406 \\
\hline \hline
\end{tabular}

La Tabla 3 muestra los valores de la métrica $C(A, B)$ para los algoritmos, la cual permite apreciar que NSGA II tiene mejores soluciones que SPEA II, aunque no todos los individuos de SPEA II son dominados por los individuos de NSGA II.

Tabla 3: Valores para la métrica $C(A, B)$

\begin{tabular}{ccc}
\hline \hline $\mathrm{A}$ & $\mathrm{B}$ & $\mathrm{C}(\mathrm{A}, \mathrm{B})$ \\
\hline NSGA II & SPEA II & 2,21 \\
SPEA II & NSGA II & 0,20 \\
\hline \hline
\end{tabular}

La Figura 2 muestra los frentes de Pareto obtenidos con los dos algoritmos implementados.

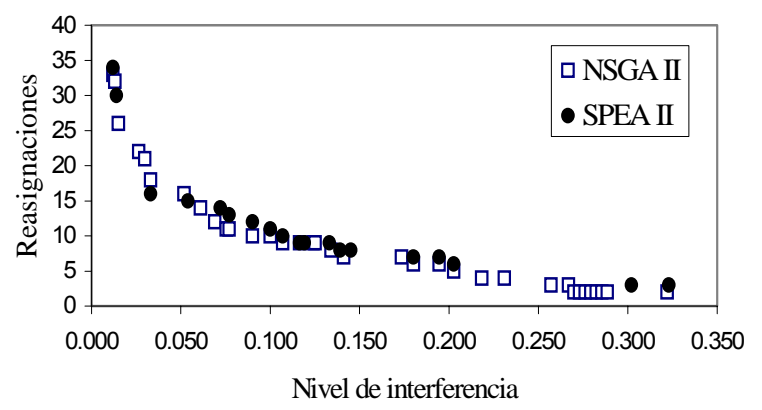

Fig. 2. Frentes de Pareto obtenidos con NSGA II y SPEA II

Además del desempeño después del proceso evolutivo, se analizó la actuación de SPEA II y NSGA II durante la evolución. Las Figuras 3 y 4 muestran la evolución de las métricas $S(A)$ y $D(A)$, respectivamente, con respecto al número de generación. NSGA II muestra una convergencia más rápida y un desempeño más estable que SPEA II. Estos resultados muestran que NSGA II no solo encuentra mejores soluciones, sino que también las encuentra de manera más rápida y confiable. 


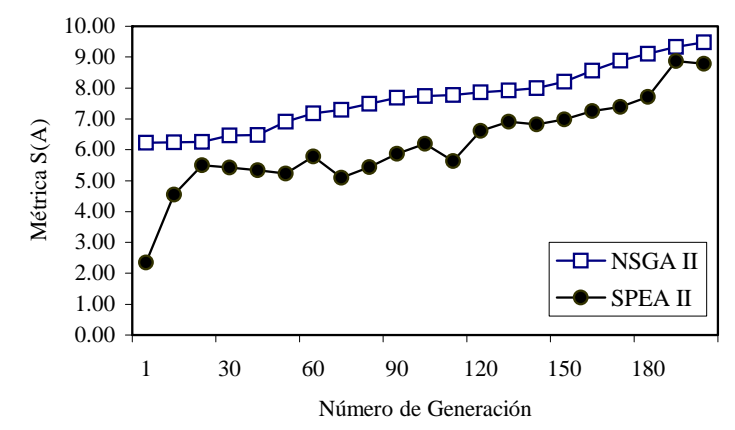

Fig. 3: Evolución de la métrica $S(A)$ con respecto al número de generación para NSGA II y SPEA II

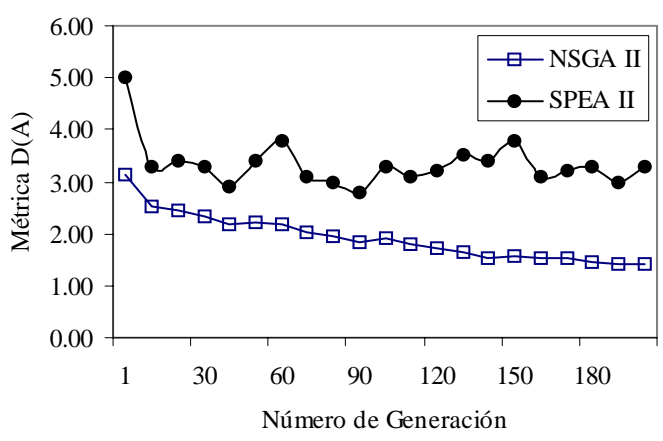

Fig. 4: Evolución de la métrica $D(A)$ con respecto al número de generación para NSGA II y SPEA II

\section{CONCLUSIONES}

La ventaja principal del enfoque utilizado, en contraste con la resolución de un problema de optimización simple objetivo y restringido, radica en la obtención de un conjunto de soluciones que le permite al operador del sistema la toma de decisiones con varias alternativas disponibles. De esta manera es posible optar por una de ellas conociendo inmediatamente el costo asociado a dicha decisión sin necesidad de resolver nuevamente un problema de optimización.

Se han aplicado dos algoritmos genéticos multi-objetivo al problema de la asignación de frecuencias. Si bien ambos algoritmos aplican elitismo, la superioridad de NSGA II radica en como maneja la preservación del mismo.

NSGA II provee un conjunto Pareto de buena cantidad y distribución de soluciones. SPEA II, por su parte entregó buenas soluciones aunque la mayor parte de ellas están localizadas en una zona del espacio de la función objetivo y su número es sensiblemente menor en comparación con NSGA II.

Los desempeños de los algoritmos fueron evaluados para identificar la contribución de los nuevos mecanismos. Se usaron tres métricas de desempeño cuantitativas: tamaño del espacio dominado, diferencia de cobertura de dos frentes de Pareto y no uniformidad del frente de Pareto. Las primeras dos métricas miden la convergencia del frente de Pareto, mientras que la última mide la distribución del frente. Puede apreciarse que los mecanismos de NSGA II mejoran apreciablemente la convergencia y distribución del frente de Pareto resultante. Las diferencias de desempeño también se manifiestan en la variación de las métricas con respecto al número de generación. NSGA II conduce a un mejor frente de Pareto de manera más eficiente y confiable.

La metodología propuesta puede ser aplicada a las diferentes generaciones de sistemas de comunicaciones móviles, ya que en todas ellas aparece el problema de asignación de recursos. Para cada caso particular deberá realizarse un adecuado análisis de las restricciones impuestas al problema.

\section{REFERENCIAS}

Coello Coello, C., D. Van Veldhuize y G. Lamont; Evolutionary Algorithms for Solving Multiobjective Problems. Kluwer Academic Publishers, New York (2002).

Deb, K.; "Multi-Objective Optimization Using Evolutionary Algorithms”, John Wiley \& Sons (2001).

Deb, K., A. Pratap, S. Agarwal y T. Meyarivan; "A fast elitist multiobjective genetic algorithm: NSGA II”, IEEE Transactions on Evolutionary Computation, 6(2), 182-197 (2002).

Fu, X., Y. Pan, A.G. Bourgeois y P. Fan; "A Three-Stage Heuristic Combined Genetic Algorithm Strategy to the Channel-Assignment Problem". In Proceedings of the 17th international Symposium on Parallel and Distributed Processing. IEEE Computer Society (2003). 
Islam, M.I. y A.B.M.S. Hossain; "Channel allocation of mobile cellular network based on graph theory" TENCON 2004. IEEE Region 10 Conference Volume B, 529-532 (2004).

Lima, M.., A. Araujo y A. Cesar; "Dynamic channel assignment in mobile communications based on genetic algorithms“. Personal, Indoor and Mobile Radio Communications, The 13th IEEE International Symposium on Volume 5(1), 2204-2208 (2002).

Naik, K. y D.S.L. Wei; "Call-on-hold for improving the performance of dynamic channel-assignment strategies in cellular networks" Vehicular Technology, IEEE Transactions,53(6), 1780-1793 (2004).

Sivarajan, K., R.J. McEliece y J.W. Ketchum; "Channel Assignment in Cellular Radio" in Proc. 39th IEEE Vehicular Technology Conference, 846-850 (1989).

Srinivas, N. y K. Deb; "Multiobjective optimization using nondominated sorting in genetic algorithms", Evolutionary Computational Journal, 2(3), 221-248 (1994).

Vidyarthi, G., A. Ngom e I. Stojmenovic; "A hybrid channel assignment approach using an efficient evolutionary strategy in wireless mobile networks". Vehicular Technology, IEEE Transactions, 54(5), 1887-1895 (2005).

Wang, W. y C. Rushforth; "An Adaptive Local Search Algorithm for the Channel Assignment Problem (CAP), " IEEE. Trans. Veh. Technol, 45(3), 459-466 (1996).

Whenhao S., F. Pingzhi y P. Yi; "Performance Evaluation of a Hierarchical Cellular System with Mobile Velocity-Based Bidirectional Call-Overflow Scheme", IEEE Transactions on Parallel and Distributed Systems 14(1), 72-83 (2003).

Zitzler, E., M. Laumanns y L. Thiele; SPEAIl: "Improving the strength pareto evolutionary algorithm". Technical Report 103, Swiss Federal Institute of Technology, Zurich, Switzerland (2001). 
